\section{Animating Mediums From Visuality of Superimposition to Drawings for Afterimage}

Catty Dan Zhang

UNC Charlotte

\section{INTRODUCTION}

In her book Phantasmagoria, Marina Warner states a relationship between vision and mentality that "one kind of mental image was described as 'eidetic', referring to optical experiences that are retained in the mind's eye with hallucinatory intensity. It comes from eidos, used by Aristotle for that which is seen, or 'form, shape, figure', both of something particular and of a generic kind of form, and it is related to idein, to see, and eidolon, a shape, image, spectre, or phantom, also an image in mind, a vision or fancy"1.

It was within a relatively short period of time- comparing with over a century long obsession of the aesthetics of superimposed moving sequences - that the discourse of animation in architecture has diverged its paradigm from analytical motion forms in the digital environment towards new possible optical experiences and atmospheric effects in physical spaces. Differing from the traditional cinematic model implemented in architectural design which stitches series of views through spatial organizations, recent investigations regarding these dynamic spatial effects have been largely inspired by mapping techniques, autonomous drawings, and hybrid mediums; or in other words- expanded operations on visuals. The long tradition of the spatiotemporal visual practice in forms of superimposed images, however, has taken on various trajectories transitioning from the static basis to animated implications. Historically used for capturing and representing motion, it is recognized identically as sets of frames with discrete positions, where the animation virtually emerges from the viewer's subconscious process of translation.

With the emergence of digital technology in architecture, Greg Lynn discussed in his book Animate Form two decades ago the analogy between Etienne-jules Marey's mechanical interpretation of his chronophotography study, and the numerical model of trajectory, velocity, points and curves as virtual forces for animating forms in the digital software. Captured on one negative during swift movements, Marey utilized various instruments and techniques for extracting continuous curvilinear flow forms of movements from the segmented frames. The act of geometric calculation laid a foundation for computational drawing and modeling, translating parameters into vector based virtual components that allow infinite extractions and transformative manipulations ${ }^{2}$.

Animating Mediums draws methodological inspiration from such precedents but attempts at an anti-continue effect from the superimposition, foregrounding concepts of images that are not seen only as design outcomes, but also as apparatuses that mediate vision. This inevitably refers to a parallel investigation with the same visual type of documented movements which not primarily looks at form but instead, sensation. Represented by Futurist, this trend tackles illusory movements intensified by color, stroke, and pigments, for achieving a "plastic analogy of dynamism", a term coined by Gino Severini in the 1914. The subjective expression embedded in the artworks defies relationship of form and field defined by any mathematical equation. It appears as discrete, fragmented, yet somehow integrated based on visual and psychological tectonics rather than principles of physics and geometry; a proto-digital operation on what has been widely explored now - the raster image and the pixel. 


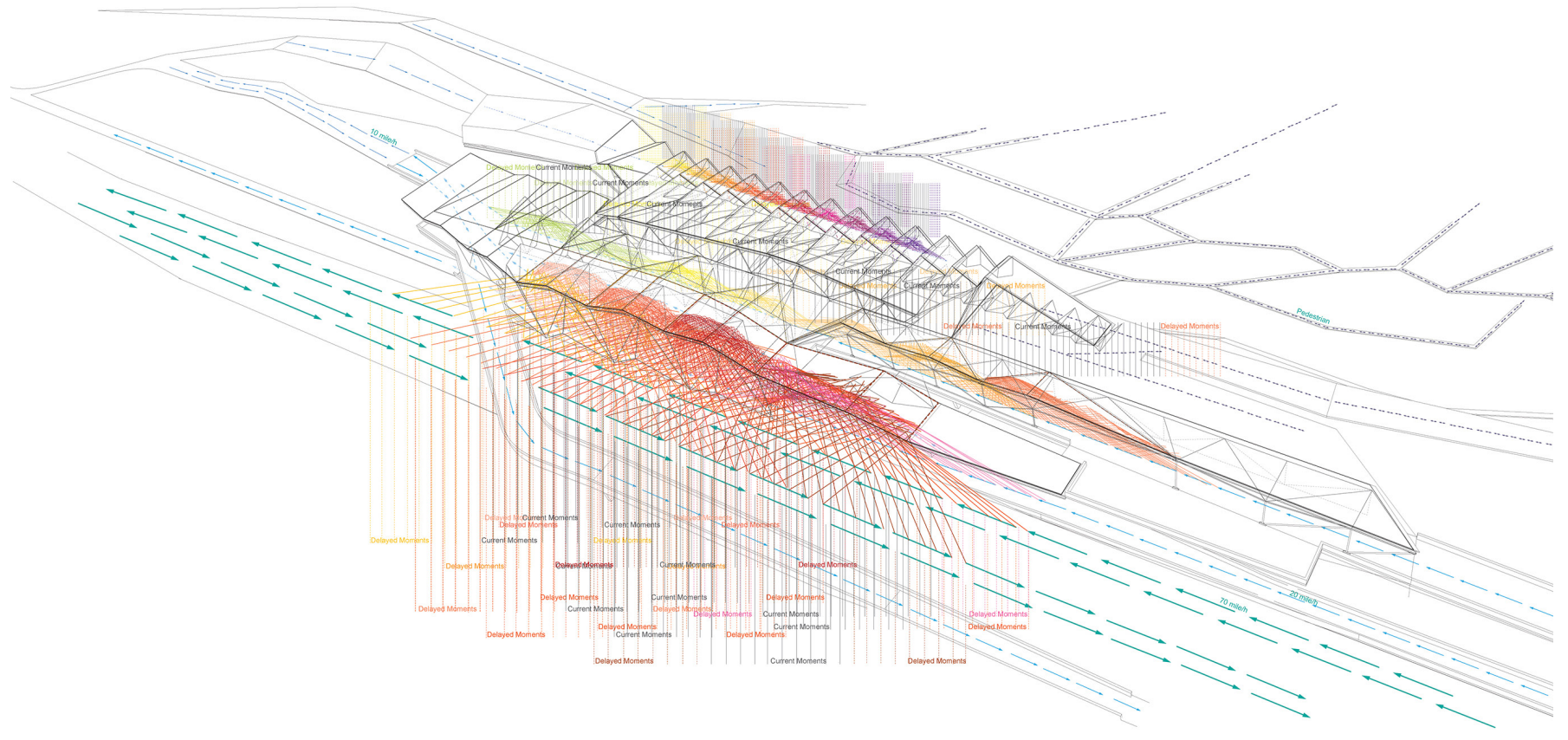

Figure 1. Delayed Moments. Spatial logics of architecture derive from mapping temporal factors that emerge from interactions between automobile lights and building geometries assorted along moving paths. Drawing by the author.

Animating Mediums investigates "the pixel" and "the animation" in two interrelated approaches: one explores the idea of "architectural pixel" as a spatial logic, while the other looks at the numerical model of pixels and the design of interactive environments using customized digital and physical tools. Both are done in forms of intensifying duplication and fragmentation- actions referring to Warner's argument on hallucinatory optical experience, as well as to Severini's statement on the plastic analogy in art forms.

\section{ARCHITECTURAL PIXEL}

Traveling at sunset on a bus ride to Marseilles from Paris, artist Brion Gysin closed his eyes as the bus entered a long enfilade of evenly spaced trees. The resultant flickering, he later wrote, swept him out of time into "a transcendental storm of color visions". The eidetic effect of experiencing the flashing of sunlight- made possible through the fleeting imagery animated by one moving along the passage at a certain speed - led to a mechanical reproduction by Gysin as the "Dreamachine" 3 . The device reassembles the condition and scenario at an infrastructural scale into a mechanism that stitches the optical illusion and the mental image. The spatial organization of the trees along the motorway, the speed of movements, and the direction of light rays, were transformed into the spinning apparatus. A cylinder with slits on the sides and a light bulb suspended in the center, is placed on a record turntable. The rotation speed allows the light to come out from the holes at a constant frequency of between 8 and 13 pulses per second, corresponding to "alpha waves", the electrical oscillations normally present in the human brain while relaxing, altering brain activity and not only the visual cortex, but the whole mind ${ }^{4}$.

It is from this narrative that I would like to propose a concept of architectural pixels. In his foreword essay for Public Intimacy, Anthony Vidler wrote, "three-dimensional space, inhabited and set in virtual motion by the body, has formed the material of modern architecture" ${ }^{\prime}$. Neither simply as narratives formulated from sequential frames nor imagery of patterns, architectural pixels could be understood as results of physical assemblies reacting to such virtual motion, so that temporal material of sliced time is converted into perceptible fragmented effects.

Imagine a set of promenades assorting movements of vehicles and pedestrians, for instance, is integrated with geometric modules that capture and retain reflections of light from automobiles moving at various speeds. As a speculative highway rest stop project (Figure 1), the design evolves around the mapping of moving speeds along planned paths. Similar to the concept of the pixel in digital imaging where it represents the smallest addressable and controllable element of a picture on the screen, the segmented experience in this architectural proposal is constructed through the practice of sampling, scaling, layering, and rotating light volumes at physical coordinates along linear axes. Individual modules - functioning as occupiable spaces - are aggregated into an assemblage of light collected and diffused from continuously moving vehicles. This spatial logic of pixels resulted in a montage of actual and delayed moments stretching along the highway; a real-time light polyphony to be experienced in motion (Figure 2).

\section{PROGRAMMED VISION}

Alongside the architectural speculation, Speed Tectonics, experimental installations developed in a seminar taught by the author, 


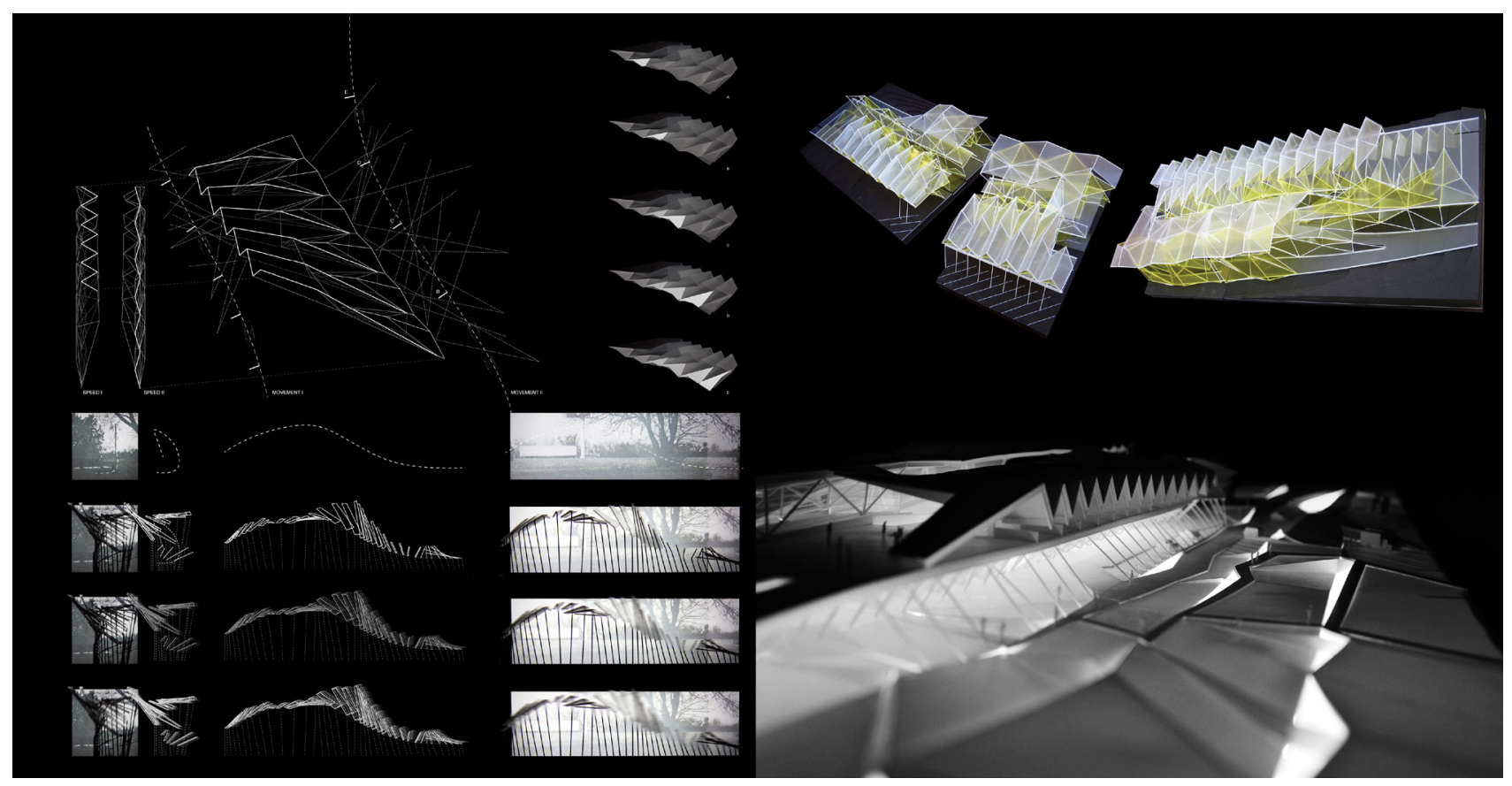

Figure 2. Activating architectural pixel sequences by fragmenting light patterns from continuously moving vehicles.

extend possibilities of mediated dynamic perception by hybridizing numerical pixels and physical mediums. Tracing analogies between the visuality of superimposition in paintings and the computational constructs of images, Speed Tectonics seeks methodological translation from Severini's concept on plastic analogies of motion, to the eidetic perception in spatial settings with human motion as inputs utilizing technology.

Employing visual methods indebted to Marey's chronophotography, the artistic techniques of tracing moving objects practiced by groups such as Futurist allows "tangible figures of physical motion" to dissolve "into abstracted and non-referential forms". This type of abstraction - directly built upon physical phenomena- intends to render perceptual experiences of motion in a plastic manner. While still appear as series of frames extracted from continuous motion, these artworks usually employ methods such as blurring fore-, middle-, and background of each frame; interlocking picture planes; as well as utilizing colored pigments. Such techniques trick human eyes which result in constant zooming and re-focusing manners, so that other sensory, as well as somatosensory are triggered by both analogy and physiology?. The final images are results from each small area on canvas differentiated from its neighbors in color, stroke pattern, textures, and so on.

The use of time-based media, on the other hand, allows the act of intensifying fragmented visual imagery to expand the physiological stimuli beyond the two-dimensional static visual format. Among pioneers in computer-animated films as such, Pixillation (1970), ENIGMA (1972), and Googoplex (1972) by Lillian F. Schwartz employing early customized computer platforms, are created with a generative process which uses an initial image and a process by which it could be transformed. These films encompass rapidly alternating abstract patterns and flicking blocks of colors which are highly dependent on saccadic motion of eyes. The resulting visual appears as a motion in space that generates a hallucinatory depth ${ }^{8}$.

Speed Tectonics combines pixels in computational processes of such visual operations with the use of pigments of discrete colors in Futurist paintings both spatially and temporally. Each set of experiments showcases the focus on a particular aspect of perceptual motion in order to unveil the linkage between the visuality of a static image and the afterimage generated by dynamic experiences. Throughout the process, customized digital tools are developed based on image processing algorithms, which are then outputted as articulated physical conditions.

\section{COLOR THRESHOLDING AND DEPTH DISTORTION}

Vertoscopes (Figure 3) is an example which explores color thresholding techniques in the digital environment and depth distortion strategies in the physical context with Street Light painted by Giacomo Balla in 1909. Featuring a blurred electric lamp overlaid by colored strokes indicating rays of light, the painting consists of segments of curves spreading out from the center of the lamp, the peaks of which are oriented in the direction towards the light source. Through an algorithmic play with colors, the animated sequence is constructed from fluctuations of pixel arrays generated from the original painting, appearing to be dynamically expanding from or contracting towards the visual center which becomes a pulsing effect. 


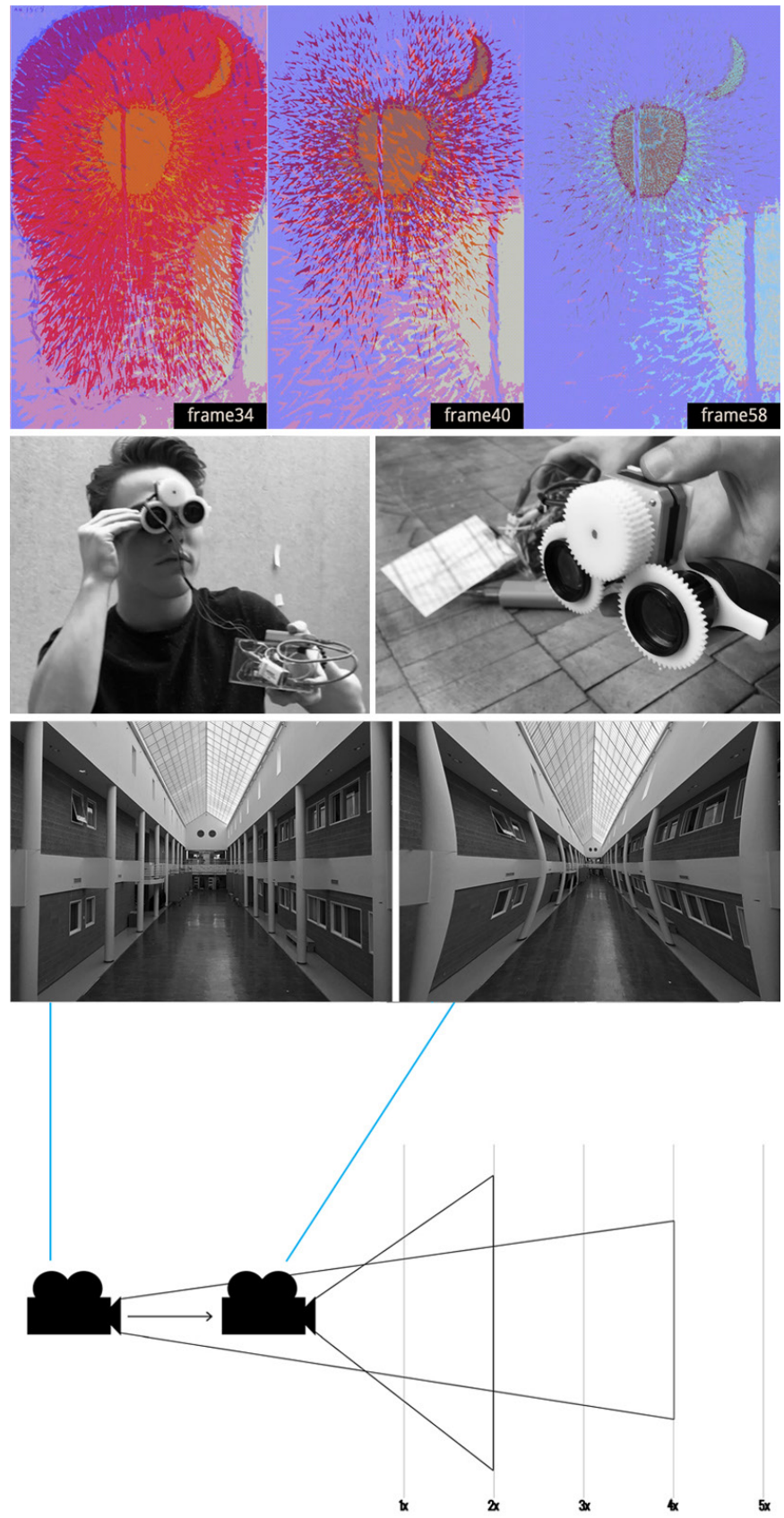

Figure 3. Vertoscopes explores color thresholding and depth distortion. (Top) selected frames from the animation of color thresholding experiments with Street Light (Balla, 1909). This project was developed in the seminar Atmospheric Animations at UNC Charlotte, Fall 2017.

The translation between computational experiments and physical space in Vertoscopes builds upon the visual dependency of misaligned layers animated at different rates between one and another. Illusion of speed or even immobility in real time- perceived on the screenis then reassembled with spatial references in the actual context of a long atrium. Through wearing an interactive eye goggle, the depth of visual field is constantly changing as one reacts to the new perception by adjusting one's own speed of movements.

\section{IMAGE TRANSFORMATION AND CONSTRUCTED GEOMETRIC REFLECTION}

Convergence of Speed (Figure 4) is another example which visually emphasizes force of motion by designing a three-dimensional system consisting of multiple axes, center points, and planes where projected animations converge towards. Experimenting with Science Against Obscurantism by Giacomo Balla in 1920 which presents a composition of convergence towards a horizontal central axis for expressing the direction and swiftness of movements, this project develops a program with a continuous scaling operation on the original painting in order to create new orders of vanishing points on the screen. While appearing to be intermittent frames extracted from continuous motion, the number of frames displayed within certain period of time, as well as the scaling factor, are a few variables that affect the effect of fragmentation and the speed of the convergence.

The transformation further takes place through constructed geometric reflection within a 6' × 6' × 6' "infinite room". With mirrors covering four sides of the walls, projections are coordinated to have dynamic interactions with corners and edges of the space as the light bounces around and generates multiplicity of imageries. The reflections allow overlaid visual portals to emerge from images moving towards designated locations with traces left behind, transforming the 6' cube into an immersive infinite network constantly distorts one's perception as one moving within the installation.

\section{COMPUTATIONAL STROKE AND DUPLICATED ARRAY}

Swifts Paths of Movement Dynamic Sequences by Giacomo Balla in 1913 is a painting composed of abstracted trajectories and frames inspired by Marey's locomotion studies. In a two-dimensional organization, seven curves in bright golden color divide the painting into several parts. They at times connect elbow joints on each frame just as what Marey did with chronophotographs the geometric interpretation of motion, while in other cases they weave through abstract patterns on separate motion paths. Experimenting with this painting, Close Encounters (Figure 5) algorithmically manipulates the type, the color pallet, and the density of strokes by sampling pixels and conducting rigorous transformations around their coordinates (Figure 6). From sparse color dots, to enlarged pixels and stretched strokes, original motion patterns are blurred, morphed, and dissolved into new abstract fields.

A thickened fog screen was designed which further stretches the computational strokes into short lines along two directions as light rays passing through the physical medium. Situated in a dark room, the array of rays are duplicated by two projectors arranged apart from each other. As one controls the interactive "painting" process with bodily gestures as inputs, the experience becomes a close encounter with three dimensional light forms gradually emerging from accumulated colored rays within the fog; a process of freezing motion within a three-dimensional volume in real time. 


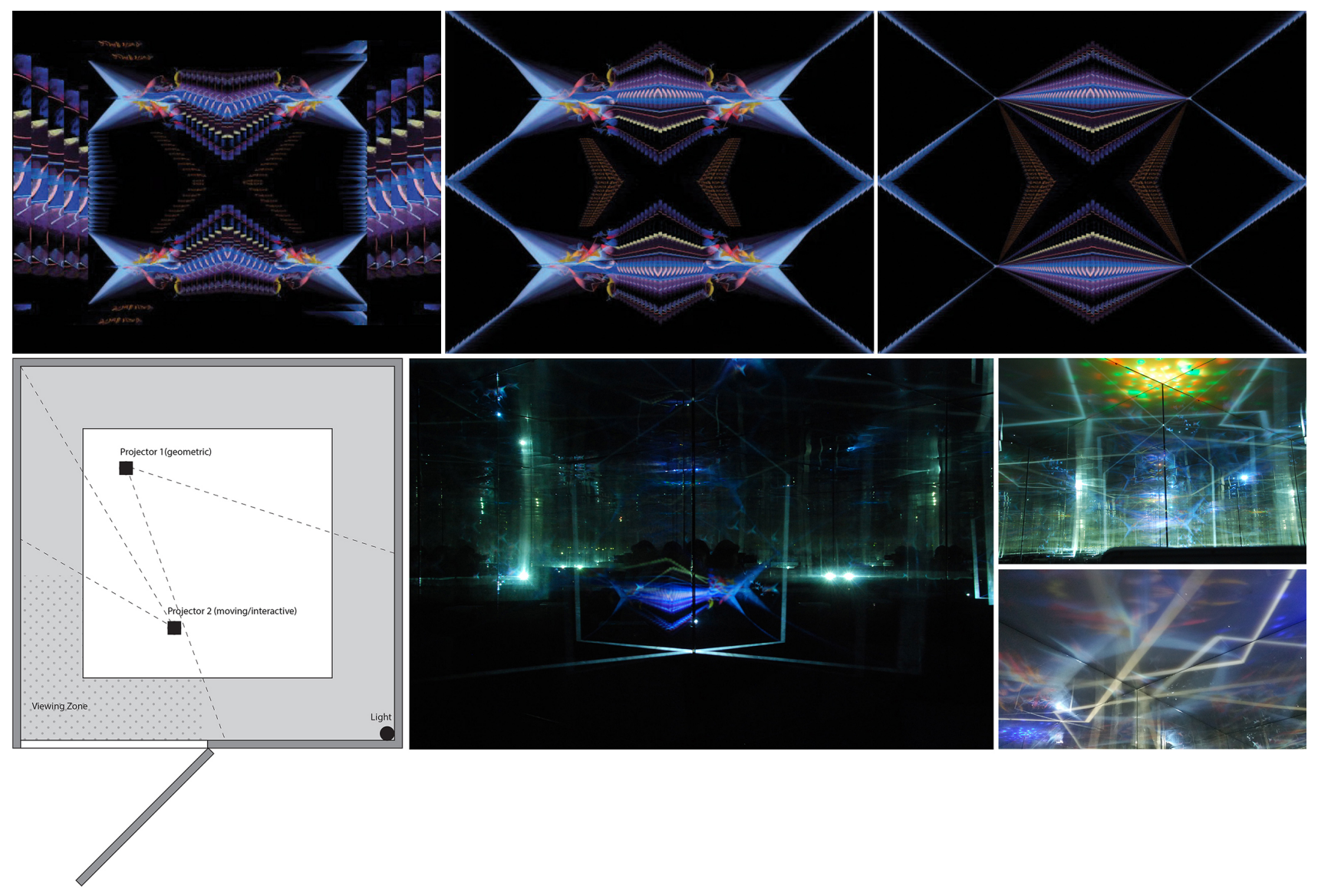

Figure 4. Convergence of Speed explores image transformation and constructed geometric reflection. (Top) Programmed image transformation studies with Science Against Obscurantism (Balla, 1920). This project was developed in the seminar Atmospheric Animations at UNC Charlotte, Fall 2017.

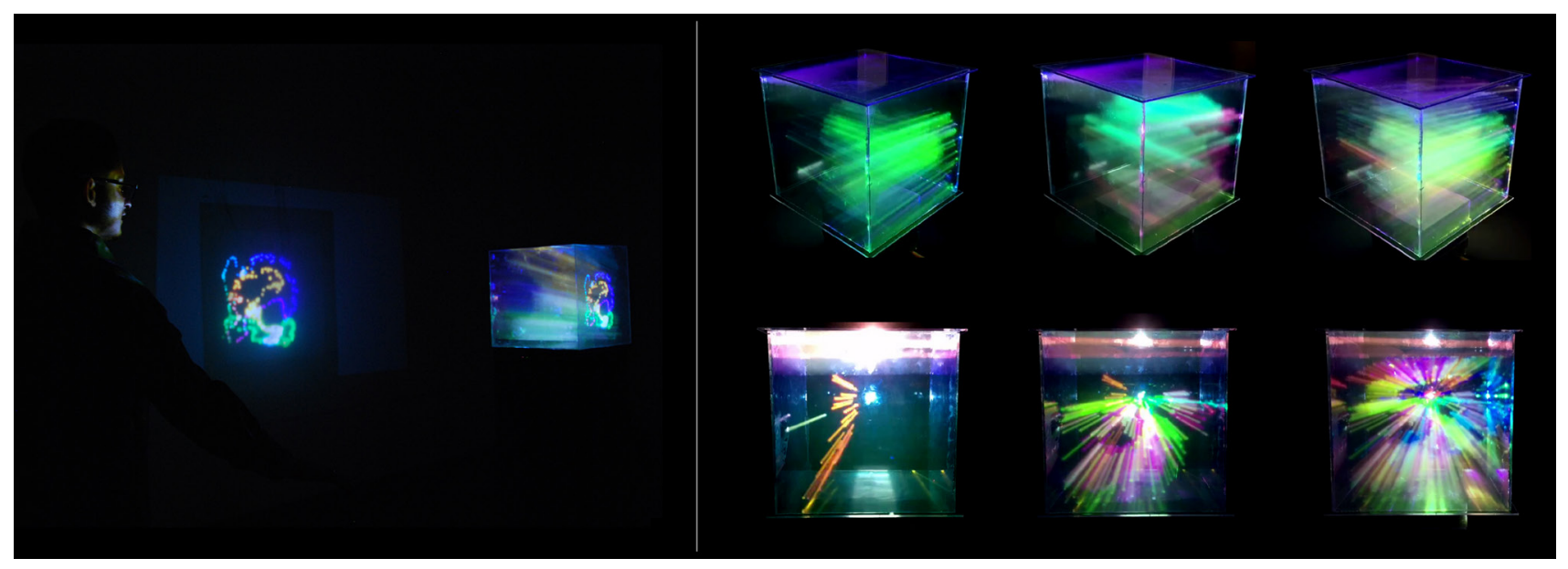

Figure 5. Close Encounters explores computational stroke and duplicated array

This project was developed in the seminar Atmospheric Animations at UNC

Charlotte, Fall 2017. 


\section{CONCLUSION}

Marey's optical apparatuses captured "the imperceptible, the fleeting, the tumultuous and the flashing of body movement in the non-digital age" 9 . Today, with digital apparatuses based on the advanced computing technology and display systems, both spatial and temporal data of swift movements could be fathomed with high accuracy and fine details. Animating Mediums explores the use of pixels and raster images as spatial logics as well as design agencies in order to uncover the experiential aspects of motion. Augmenting vision through the hybrids of physical and digital animations, Animating Mediums advances an intent of using representation concepts as design tools. Projects here question how swift movements mediate vision from a human-centric perspective, where the original superimposed frames are further animated across mediums and are related in new orders. These animations examine the possibility of spaces that actively detect, modify, and intensify motion.

\section{ACKNOWLEDGMENT}

The author would like to thank students in Atmospheric Animations: Speed Tectonics Seminar at UNC Charlotte in Fall 2017 who developed projects included in this paper: Vertoscopes by Clair Shue and Jon Warner; Convergence of Speed by Carlos Martinez and Ibha Shrestha; and Close Encounters by Mahdi Ghavidel Sedehi and Amir Vafa.

\section{Notes}

1. Marina Warner, Phantasmagoria: Spirit Visions, Metaphors, and Media into the Twenty-First Century, (Oxford, New York: Oxford University Press, 2006), 124.

2. Greg Lynn, Animate Form, (New York, NY: Princeton Architectural Press, 1999), 25-29.

3. John Geiger, Chapel of Extreme Experience: A Short History of Stroboscopic Light and the Dream Machine, (New York, NY: Soft Skull Press, 2003).

4. W. Gray Walter, The Living Brain, (New York, NY: W. W. Norton \& Company, 1963).

5. Anthony Vidler "Forward", in Giuliana Bruno, Public Intimacy, (Cambridge, MA: MIT Press, 2007), ix.

6. David Mather "Analogies", in Caroline Jones, David Mather and Rebecca Uchill (eds.), Experience: Culture, Cognition, and the Common Sense, (Cambridge, MA: MIT Press, 2016), 57.

7. Ibid., 62.

8. Zabet Patterson, Peripheral Vision: Bell Labs, the S-C 4020, and the Origins of Computer Art, (Cambridge, MA: MIT Press, 2015), 85-105.

9. Chris Salter, Entangled: Technology and the Transformation of Performance, (Cambridge, MA: MIT Press, 2010).
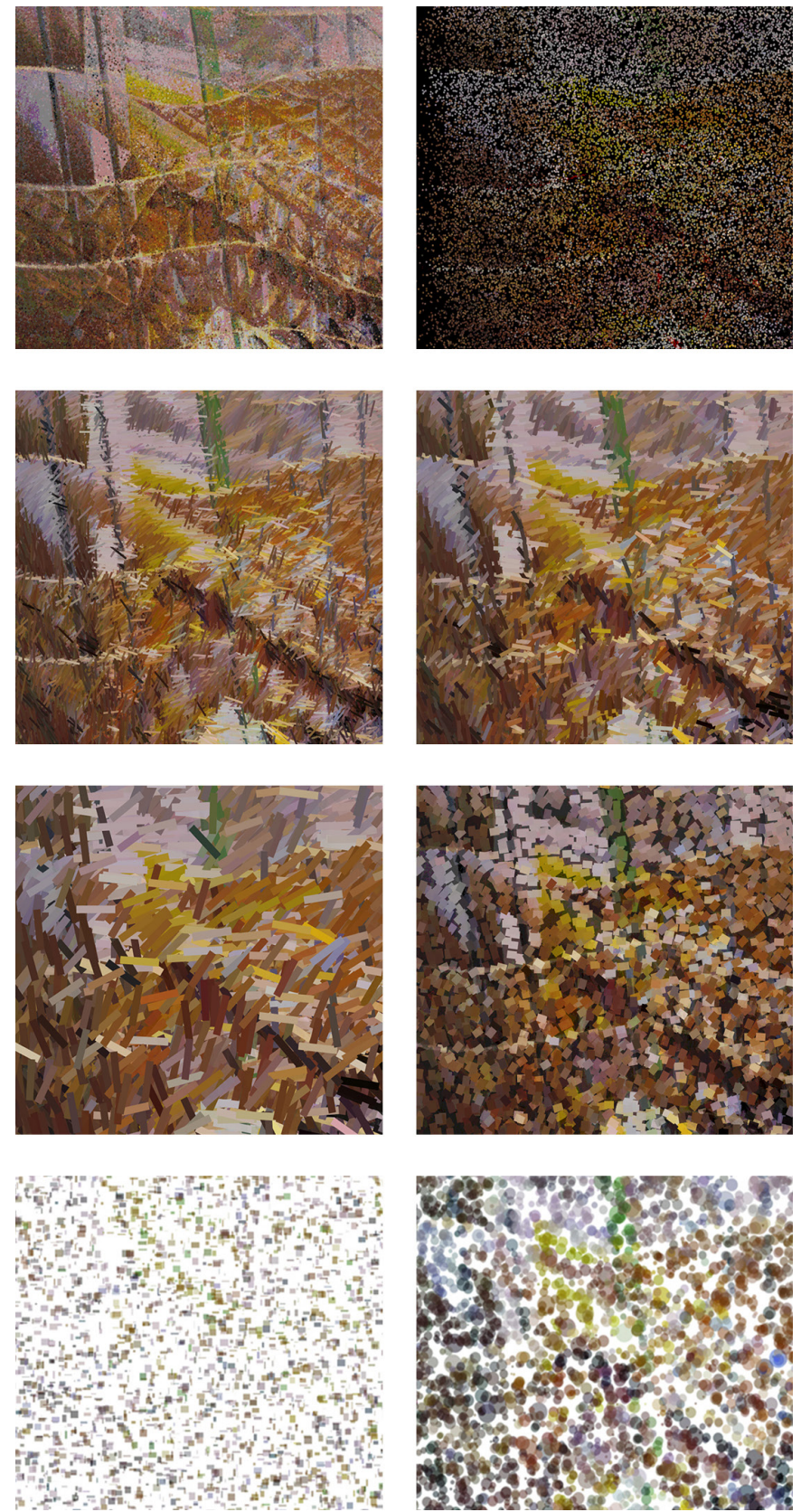

Figure 6. Selected computational stroke experiments with Swifts Paths of Movement Dynamic Sequences (Giacomo Balla, 1913) for project Close Encounters. 